\title{
Changes in Day 1 Post-Operative Intraocular Pressure Following Sutureless 23-Gauge and Conventional 20-Gauge Pars Plana Vitrectomy
}

\author{
Emily Gosse ${ }^{*, 1}$, Richard Newsom ${ }^{2}$, Peter Hall ${ }^{3}$ and Jonathan Lochhead ${ }^{4}$ \\ ${ }^{I}$ Department of Ophthalmology, St Mary's Hospital, Newport, Isle of Wight, UK \\ ${ }^{2}$ Department of Ophthalmology, Southampton General Hospital, Southampton, UK \\ ${ }^{3}$ University of Leeds, Leeds, UK \\ ${ }^{4}$ Department of Ophthalmology, St Mary's Hospital, Newport, Isle of Wight, UK
}

\begin{abstract}
Background/Aims: The benefits associated with transconjunctival sutureless vitrectomy techniques are continuing to be defined. The purpose of this study was to compare the incidence of extreme changes in day 1 intraocular pressure (IOP) following 23-gauge sutureless vitrectomy compared with conventional 20-gauge vitrectomy.

Methods: Fifty consecutive 23-gauge and 50 consecutive 20-gauge cases were included; eyes with a history of previous vitreoretinal surgery were excluded. 23-gauge surgery was completed with passive fluid air exchange where no long acting tamponade was indicated. The surgery remained sutureless unless a leak was visible at the end of the procedure. Data were collected by retrospective case note review. Statistical analysis was carried out using Fisher's exact and chisquare tests.

Results: Twenty-two percent (11/50) of 23-gauge vitrectomies required suturing of one or more ports. On the first postoperative day hypotony (IOP $<5 \mathrm{mmHg}$ ) occurred in $1 / 50$ eyes in the 20- and 23-gauge groups. Raised pressure (IOP $>21 \mathrm{mmHg}$ ) occurred in $30 \%(15 / 50)$ of eyes in the 20-gauge group and 8\% (4/50) of eyes in the 23-gauge group; IOP> $30 \mathrm{mmHg}$ only occurred in the 20 -gauge group (3/50).

Conclusion: Fluid air exchange following 23-gauge vitrectomy is associated with very low risk of day 1 hypotony. This predominantly sutureless technique appears to reduce the incidence and magnitude of early post-operative IOP elevation compared with conventional 20-gauge vitrectomy.
\end{abstract}

Keywords: Transconjunctival sutureless vitrectomy, 23-gauge vitrectomy, post-operative hypotony, post-operative IOP.

\section{INTRODUCTION}

Transconjunctival sutureless vitrectomy (TSV) techniques are reported to offer considerable potential benefits when compared to conventional pars plana vitrectomy [1]. Transconjunctival sutureless vitrectomy was first described by Chen 1996 [2]. In 2005 Fujii et al. [3] introduced the 25-gauge system. Eckardt [4] introduced the 23 -gauge system in 2005 . These techniques have been widely adopted by the vitreoretinal community. As with any novel technique, new surgical challenges have been encountered and a new profile of complications described. The 23-gauge system has some potential advantages when compared to the 25 -gauge system. The greater diameter instruments are more robust than the 25-gauge system, providing more familiar handling and a smoother transition for surgeons trained in conventional 20-gauge surgery. The increased instrument gauge also allows a slightly higher flow rate in comparison to the 25 -gauge system. In addition the range of instruments is currently more diverse and the use of

*Address correspondence to this author at the Department of Ophthalmology, St Mary's Hospital, Newport, Isle of Wight, PO30 5TG, UK; Tel: 01983 534504; Fax: 01983 534415;

E-mail: accountmailing@yahoo.co.uk silicone oil more practical in a 23-gauge system. The purpose of this study was to compare the incidence of extreme changes in intra-ocular pressure (IOP) in conventional sutured 20-gauge surgery with that of 'modified' sutureless 23 -gauge vitrectomy in the early postoperative period (Day 1 IOP). Pre-operative and late changes in IOP were not included as part of this study.

\section{MATERIALS AND METHODOLOGY}

Data were collected by retrospective case note review. Fifty consecutive 20-gauge cases, followed by 50 consecutive 23-gauge cases, in whom elective vitrectomy surgery was indicated for the management of epiretinal membrane (ERM), vitreomacular traction (VMT) or macular hole were included in the study. Patients with a history of previous vitreoretinal surgery to the same eye were excluded.

Each patient underwent standard 3-port pars plana vitrectomy. All surgery was performed by two experienced consultant vitreo-retinal surgeons (JL and $\mathrm{RN}$ ) in one centre using the Alcon Accurus and Alcon 20- and 23-gauge systems. The cut rate was controlled by the surgeon between 1500 - 2500 cuts per minute. Where indicated clinically, combined phacovitrectomy was performed. 
23-gauge wound construction was in two steps, initially oblique and then perpendicular to the sclera (following lateral displacement of the conjunctiva). Conventional suturing of the ports was carried out for all patients in the 20gauge group. 23-gauge surgery was sutureless unless an obvious leak was present at the end of the surgery; in such cases ports were sutured with 7'0 Vicryl and the number of sutures documented. Leakage was identified by the observation of small bubbles escaping from the sclerostomy port. Fluid air exchange (FAX) was carried out at the end of all 23-gauge procedures (100\% air fill) where gas and oil tamponade were not indicated. This is currently not considered conventional practise and is therefore referred to as a 'modification' throughout this text. Patients were examined on day 1 at the slit lamp and all IOP measurements were made using Goldmann applanation tonometry. Hypotony was defined as IOP $<5 \mathrm{mmHg}$ on day 1 . Patients with raised pressure on day 1 were treated, where indicated clinically, with topical hypotensives (timolol/dorzolamide combination) and oral acetazolamide if required.

Statistical analysis was carried out using Fisher's exact test and chi-square test. The odds ratio for day 1 IOP greater than $21 \mathrm{mmHg}$ was adjusted for the use of C3F8 gas using logistic regression. For 12 cases the Goldmann IOP was recorded as "normal" in the case notes (normal range 5 $21 \mathrm{mmHg}$ ) but no numerical value was noted. For these cases the IOP was assumed to be equal to the mean of all normal IOP measurements $(15 \mathrm{mmHg})$. For comparison, the odds ratio was also calculated after the cases without numerical IOP values were excluded. The analysis was implemented in the $\mathrm{R}$ statistical programming language [5].

Age distribution at the time of surgery was 57-85 years (mean $73+/-8.9$ ) in the 23-gauge group and 48 - 88 years (mean $70+/-7.2$ ) in the 20 -gauge group. There were 21 male eyes and 29 female eyes in the 23-gauge group, 16 male and 34 female eyes in the 20-gauge group.

Twenty-six patients were pseudophakic prior to surgery (14/50 (28\%) in the 23-gauge group, 12/50 (24\%) in the 20gauge group. Thirty-three cases were combined procedures with phacoemulsification with IOL (18/50 (36\%) in the 23gauge group, $15 / 50(30 \%)$ in the 20 -gauge group). A peel was performed in 86 cases (43/50 in the 23-gauge group, $43 / 50$ in the 20-gauge group). Those not peeled were predominantly cases of VMT in addition to 5 cases of Stage 2 macular hole (Table $\mathbf{1}$ ).

Fifty-five cases underwent macular hole repair (24/50 in the 23-gauge group, $31 / 50$ in the 20-gauge group). Thirty-six cases underwent surgery for epiretinal membrane (21/50 in the 23-gauge group, $15 / 50$ in the 20 -gauge group). Nine patients were specifically identified as having purely vitreomacular traction pre-operatively $(5 / 50$ in the 23 -gauge group, 4/50 in the 20-gauge group) (Table 1).

\section{RESULTS}

Mean pre-op visual acuity in the 23-gauge group was 0.67 (SD+/-0.35) LogMAR units; mean post-operative visual acuity at 2 months was 0.44 (SD+/-0.34) LogMAR units. In the 20-gauge group mean pre-operative visual acuity was 0.77 (SD+/-0.36) LogMAR units; mean post-operative visual acuity at 2 months was 0.55 (SD+/-0.37) LogMAR units.
Therefore the mean gain in visual acuity (measured 2 months post-operatively) was 0.23 LogMAR units in the 23-gauge group and 0.22 LogMAR units in the 20-gauge group. This difference was not statistically significant $(\mathrm{p}=0.79)$.

Table 1. Patient Demographic Data for the 20- and 23-Gauge Groups

\begin{tabular}{|l|c|c|c|}
\hline & $\begin{array}{c}\text { 20-Gauge } \\
(\mathbf{n = 5 0})\end{array}$ & $\begin{array}{c}\text { 23-Gauge } \\
(\mathbf{n}=50)\end{array}$ & p-Value \\
\hline \hline Age (years) & $\begin{array}{c}70+/-8.9 \\
(\text { range } 48-88)\end{array}$ & $\begin{array}{c}73+/-7.2 \\
(\text { range, 57-85) }\end{array}$ & $\begin{array}{c}0.067 \\
\text { (t-test) }\end{array}$ \\
\hline Sex (Male/Female) & $16: 34$ & $21: 29$ & 0.107 \\
\hline Eye (OD/OS) & $23: 27$ & $26: 24$ & 0.689 \\
\hline Anaesthetic (GA/LA) & $32: 18$ & $29: 21$ & 0.682 \\
\hline Pseudophakic & $12 / 50(24 \%)$ & $14 / 50(28 \%)$ & 0.820 \\
\hline Combined Procedure & $15 / 50(30 \%)$ & $18 / 50(36 \%)$ & 0.670 \\
\hline ILM or ERM Peel & $43 / 50(86 \%)$ & $43 / 50(86 \%)$ & 1.000 \\
\hline Surgery for macular hole & $31 / 50(62 \%)$ & $24 / 50(48 \%)$ & 0.848 \\
\hline Surgery for epiretinal membrane & $15 / 50(30 \%)$ & $21 / 50(42 \%)$ & 0.298 \\
\hline Surgery for vitreomacular traction & $4 / 50(8 \%)$ & $5 / 50(10 \%)$ & 1.000 \\
\hline
\end{tabular}

Macular hole closure rates (with one procedure, measured at 2 month follow-up) were 96\% (23/24) in the 23gauge group and $90 \%(28 / 31)$ in the 20-gauge group. This difference was not statistically significant $(p=0.62)$. All those that failed to close were classified as Stage 4 pre-operatively.

Ten cases (4/50 in the 23-gauge group, 6/50 in the 20gauge group) required further retinal procedures. Three ERM cases ( 2 in the 23 -gauge and 1 in the 20 -gauge group) required a further peel for ERM recurrence and metamorphopsia. Three patients underwent further macular hole surgery ( 1 in the 23-gauge and 2 in the 20-gauge group). Two patients in the 20 -gauge group required removal of silicone oil. One patient undergoing vitrectomy for VMT developed a full thickness macular hole post-operatively and required surgery. One patient in the 20-gauge group developed post-operative retinal detachment requiring further vitrectomy, cryotherapy and gas tamponade. No patient in either study group developed post-operative endophthalmitis.

Mean day 1 IOP was $15 \mathrm{mmHg} \mathrm{SD}+/-5.3$ (range 3-30) in the 23-gauge group compared to $21 \mathrm{mmHg} \mathrm{SD}+/-6.8$ (range $3-40)$ in the 20-gauge group. This difference was statistically significant $(\mathrm{p}<0.0001)$.

Hypotony (IOP<5) occurred in $2 \%(1 / 50)$ eyes in the 23 gauge group and $2 \%(1 / 50)$ eyes in the 20-gauge group $(p=1.000)$. The former occurred following a combined phacovitrecomy for macular hole with $16 \%$ C3F8 gas tamponade. One port was sutured. The latter occurred in a pseudophake undergoing vitrectomy and peel (with air) for ERM. In both cases hypotony resolved spontaneously without intervention or associated complications.

Raised pressure (IOP > $21 \mathrm{mmHg}$ ) occurred in $30 \%$ (15/50) 20-gauge eyes and 8\% (4/50) 23-gauge eyes $(\mathrm{p}=0.005)$. IOP $>30 \mathrm{mmHg}$ was only observed in the 20 - 


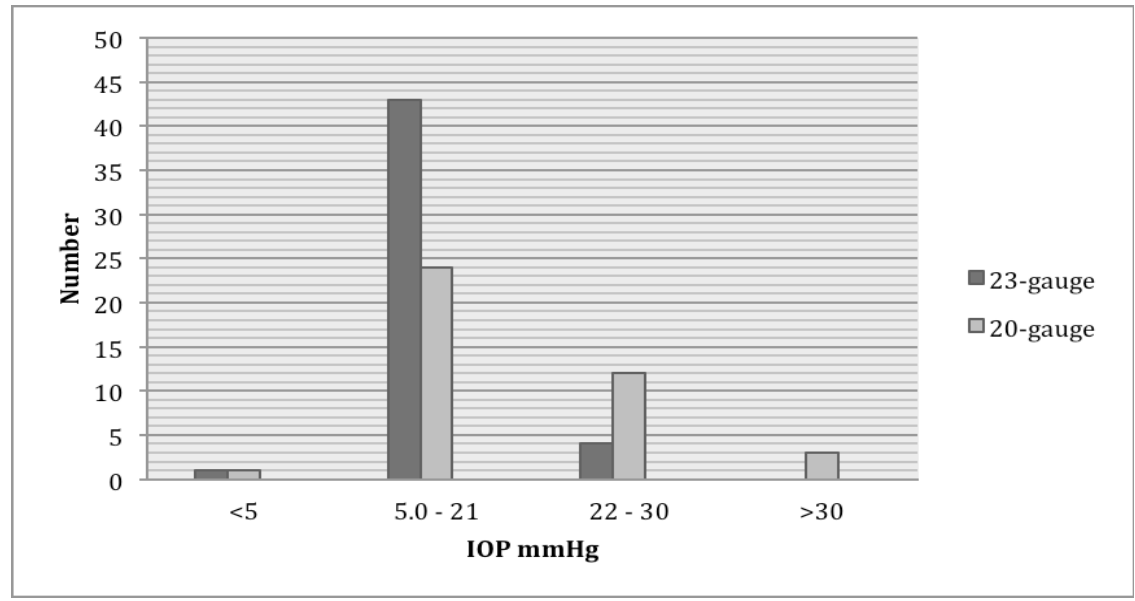

Fig. (1). Day 1 intraocular pressure (IOP $\mathrm{mmHg}$ ) in the 20- and 23-gauge groups.

gauge group (3/50) (Fig. 1). Overall there were significantly more eyes without an extreme change in post-operative IOP in the 23 -gauge $(86 \%, 43 / 50)$ compared to the 20 -gauge group $(48 \%, 24 / 50)$.

Twenty-two percent (11/50) of eyes in the 23-gauge group required suturing of one or more ports. Eight eyes received 1 suture, 3 eyes required 3 sutures (Table 2 ). Patients in the 20-gauge group had all three ports sutured as standard.

Table 2. Number of Sutures Required to Close Ports in the 23-Gauge Group Compared to Day-1 IOP

\begin{tabular}{|c|c|c|}
\hline Number of Sutures & Number of Eyes $(\mathbf{n}=\mathbf{5 0})$ & Mean IOP $(\mathbf{m m H g})$ \\
\hline \hline 0 & $78 \%(\mathrm{n}=39)$ & $16(6-30)$ \\
\hline 1 & $16 \%(\mathrm{n}=8)$ & $12(3-20)$ \\
\hline 2 & $0 \%(\mathrm{n}=0)$ & - \\
\hline 3 & $6 \%(\mathrm{n}=3)$ & $16(8-20)$ \\
\hline
\end{tabular}

All cases (19 eyes) in the 20- and 23-gauge groups with an elevated IOP $(>21 \mathrm{mmHg})$ occurred following insertion of $\mathrm{C} 3 \mathrm{~F} 8$ gas (concentration ranging from 14-18\%). C3F8 was used in $44 \%(22 / 50)$ eyes in the 23-gauge group and $68 \%$ $(34 / 50)$ of eyes in the 20-gauge group $(\mathrm{p}=0.0262)$ (Table 3$)$.

Table 3. Intraocular Tamponade Used in the 20- and 23Gauge Groups

\begin{tabular}{|c|c|c|c|c|}
\hline Tamponade & $\begin{array}{c}\text { Concentration } \\
(\text { Range })\end{array}$ & $\begin{array}{c}\mathbf{2 3 - G a u g e} \\
(\mathbf{n}=\mathbf{5 0})\end{array}$ & $\begin{array}{c}\mathbf{2 0 - G a u g e} \\
(\mathbf{n}=\mathbf{5 0})\end{array}$ & p-Value \\
\hline \hline Air & $100 \%$ & $44 \%(\mathrm{n}=22)$ & $16 \%(\mathrm{n}=8)$ & $0.0041^{*}$ \\
\hline SF6 & $20 \%$ & $10 \%(\mathrm{n}=5)$ & $8 \%(\mathrm{n}=4)$ & 1.0000 \\
\hline C3F8 & $14-20 \% \dagger$ & $44 \%(\mathrm{n}=2)$ & $68 \%(\mathrm{n}=34)$ & $0.0262 *$ \\
\hline Silicone Oil & $100 \%$ & $2 \%(\mathrm{n}=1)$ & $8 \%(\mathrm{n}=4)$ & 0.3622 \\
\hline
\end{tabular}

$\dagger$ mean C3F8 concentration $15.37 \%$ in 20-gauge group vs $16.8 \%$ in 23 -gauge group $\mathrm{p}=0.0004$.

The unadjusted odds ratio for raised IOP $(>21 \mathrm{mmHg})$ in those treated with 23-gauge vitrectomy compared with those treated with 20 -gauge vitrectomy was 0.20 (95\% confidence intervals $0.05-0.62 ; \mathrm{p}=0.008)$. The odds ratio for raised IOP adjusted for C3F8 use was 0.26 (95\% confidence intervals 0.07 - 0.86; $\mathrm{p}=0.037)$. Twelve patients had no numerical value assigned to their IOP (which was recorded as "normal" in the case notes). For these cases the IOP was assumed to be equal to the mean of all normal IOP measurements $(15 \mathrm{mmHg})$. Excluding these 12 cases with missing numerical IOP values gives the unadjusted odds ratio for raised IOP $(>21 \mathrm{mmHg}$ ) in those treated with 23 gauge vitrectomy compared with those treated with 20 gauge vitrectomy was 0.15 (95\% confidence intervals $0.04-0.47 ; p$ $=0.002$ ). The odds ratio for raised IOP adjusted for C3F8 use was $0.20 \quad(95 \%$ confidence intervals 0.05 - 0.68 ; $\mathrm{p}=0.015$ ).

In the 20-gauge group $16 \%(8 / 50)$ cases had intraocular tamponade with air, $8 \%$ (4/50) with SF6, 68\% (34/50) with C3F8 and $8 \%(4 / 50)$ with silicone oil. Silicone oil was required in four cases because of the presence of retinal breaks with size and location requiring long acting tamponade. In the 23-gauge group 44\% (22/50) cases had intraocular tamponade with air, 10\% (5/50) with SF6, 44\% (22/50) with C3F8 and 2\% (1/50) with silicone oil. Silicone oil was used in one case for the treatment of a longstanding macular hole (Table 3). Table 4 shows the type of intraocular tamponade used in each group, compared to Day 1 IOP.

Table 4. Intraocular Tamponade Used in the 20- and 23Gauge Groups, Compared to Mean Day 1 IOP

\begin{tabular}{|c|c|c|c|}
\hline \multirow{2}{*}{ Tamponade } & \multicolumn{2}{|c|}{ Mean Day 1 IOP $(\mathbf{m m H g})$} & \multirow{2}{*}{ p-Value } \\
\cline { 2 - 3 } & $\mathbf{2 3 - G a u g e}$ & $\mathbf{2 0 - G a u g e}$ & \\
\cline { 2 - 3 } & $(\mathbf{n}=\mathbf{5 0})$ & $(\mathbf{n}=\mathbf{5 0})$ & \\
\hline \hline Air & $13(\mathrm{n}=22)$ & $13(\mathrm{n}=8)$ & 1.0000 \\
\hline SF6 & $18(\mathrm{n}=5)$ & $20(\mathrm{n}=4)$ & 0.0993 \\
\hline C3F8 & $17(\mathrm{n}=22)$ & $23(\mathrm{n}=34)$ & 0.0035 \\
\hline Silicone Oil & $10(\mathrm{n}=1)$ & $15(\mathrm{n}=4)$ & - \\
\hline
\end{tabular}

Mean IOP in the 20-gauge group was $23 \mathrm{mmHg}$ following combined phacovitrectomy and $19 \mathrm{mmHg}$ 
following vitrectomy alone $(\mathrm{p}=0.06)$. Mean IOP in the 23gauge group was $17 \mathrm{mmHg}$ following combined phacovitrectomy and $14 \mathrm{mmHg}$ following vitrectomy alone $(\mathrm{p}=0.07)$. Eighty-nine percent $(17 / 19)$ of eyes with an IOP greater than $21 \mathrm{mmHg}$ occurred following macular hole surgery.

\section{DISCUSSION}

Changes in IOP following vitrectomy are a well recognised phenomenon [6], both in the immediate and late post-operative period. Raised pressure is a potentially serious complication following conventional 20-gauge surgery. In contrast, initial reports have identified concerns regarding the rates of post-operative wound leak, hypotony and endophthalmitis associated with the emerging transconjunctival sutureless vitrectomy techniques [7-10]. Current evidence, however, suggests a comparable safety profile when compared to "gold standard" conventional 20gauge surgery [11-15]. The purpose of this study was to compare the incidence of extreme changes in IOP following 'modified' 23-gauge surgery compared to the conventional 20 -gauge technique in the immediate post-operative period.

Initial reports have suggested an increased risk of postoperative hypotony and wound leak immediately following transconjunctival sutureless vitrectomy techniques $[8,16]$. In our study hypotony (IOP $<5 \mathrm{mmHg}$ ) was uncommon in both groups and was not more frequent in the 23-gauge group (2\% (1/50) eyes in the 23-gauge group and $2 \%(1 / 50)$ eyes in the 20-gauge group). The hypotonous eye in the 23 -gauge group received a suture to 1 port (the eye in the 20-gauge group received three standard sutures). In both cases hypotony resolved spontaneously without intervention or associated complications. A recent study showed a similar rate of day 1 post-operative hypotony to our study, following sutureless 23 -gauge surgery (3\%); in contrast to our study, the rate of hypotony in the 20-gauge group was statistically significantly lower $(0 \% \mathrm{p}<0.001)$ [17]. In papers where the rate of hypotony is reported to be higher $(9.7 \%$ day 1 IOP $<5 \mathrm{mmHg}$ ), it has been observed to resolve spontaneously within the first week [18].

The rate of sclerotomy leakage, significant enough to require suturing, has been reported to occur in over one third of transconjunctival sutureless vitrectomy cases [19]. The low rate of hypotony in our study was achieved using a predominantly sutureless technique. Twenty-two percent (11/50) of eyes in the 23-gauge group required suturing; $73 \%(8 / 11)$ of these received only one suture, $27 \%(3 / 11)$ required all 3 ports to be sutured. Careful assessment of the wound at the end of surgery allows any obvious leak to be rectified with the judicious use of sutures. Some studies have suggested that wound construction can influence leakage and subsequent hypotony [20,21]. We believe that, in addition to meticulous wound construction and appropriate suture usage, fluid-air exchange is vital step in reducing the rate of postoperative hypotony and it's associated complications. It is thought that this technique effectively plugs the inner aspect of the ports with remnants of vitreous base, sealing them internally and thereby preventing leakage [22]. It is also possible that the increased surface tension of air, compared to fluid, helps to create a seal that reduces wound leak. Fluid-air exchange also allows leaks to be immediately and easily identified (small bubbles can easily be observed escaping from the leaking sclerotomy port) compared to the often quite subtle appearance of BSS leakage. An interesting prospective study by Ho et al. found no difference in postoperative IOP in fluid versus air filled eyes [23], whereas another recent study found that Day 1 IOP was significantly higher in gas filled eyes compared to fluid filled eyes [24].

Our study appears to show a reduction in the incidence and magnitude of post-operative IOP changes when compared to conventional 20-gauge vitrectomy. Raised IOP on day 1 following surgery was significantly more common in the 20-gauge compared to the 23-gauge group. Extreme changes in post-operative IOP $(>30 \mathrm{mmHg})$ were entirely limited to the 20-gauge group.

Over all this data appears to show that raised postoperative IOP is less common in the 23-gauge group when compared to the 20-gauge group. Other studies have indicated a lower risk of raised IOP following 23-gauge surgery but also reported a higher rate of hypotony than we observed [16]. We believe that the 23-gauge port acts as a one-way valve allowing the release of a small amount of air when IOP increases significantly. This hypothesis is speculative but does appear to be visible in practise during the final stages of vitrectomy surgery. Very high IOP at the conclusion of Air-Gas exchange and removal of ports is generally accompanied by venting of small amounts of gas subconjunctivally until IOP approaches a more normal range.

In both groups all eyes with an elevated IOP $(>21 \mathrm{mmHg})$ occurred following insertion of C3F8 gas (concentration ranging from 14-18\%). Expansile gases, by their nature, are capable of generating an increase in intra-ocular pressure although this would not be expected on the first postoperative day. The gas fill was assessed in all eyes on day one and found to be less than or equal to $80 \%$ in all cases. Reports in the literature have suggested intra-ocular tamponade with gas, rather than fluid, does increase the risk of post-operative pressure spikes [25]. In particular, this spike is reported to occur at 24 hours post surgery following conventional 20-gauge vitrectomy [26]. Intra-ocular tamponade with C3F8 was more common in the 20-gauge compared to the 23 -gauge group $(68 \%, 34 / 50$, compared to $44 \%, 22 / 50)$. Using logistic regression analysis, we were able to show that, even allowing for the increased use of C3F8 in the 20-gauge group, extreme elevation in IOP was more common in 20-gauge compared to 23-gauge surgery. The use of $\mathrm{C} 3 \mathrm{~F} 8$ therefore seems to be associated with increased IOP postoperatively in both groups. Importantly the magnitude of the pressure rise was less in the 23-gauge than the 20-gauge group despite the use of a higher mean concentration of $\mathrm{C} 3 \mathrm{~F} 8$ in the 23-gauge group.

Eighty-nine percent (17/19) of eyes with an IOP $>21 \mathrm{mmHg}$ occurred following macular hole surgery. This correlates with the use of expansile gas (most commonly C3F8) during macular hole surgery, compared to fluid air exchange following vitrectomy for epiretinal membrane. Importantly there was not a significant difference between the two groups in the number of eyes undergoing surgery for macular hole repair. 
There was no statistically significant difference between the mean Day 1 IOP for patients undergoing vitrectomy or combined phacovitrectomy in either the 20 - or the 23 -gauge groups.

Post-operative endophthalmitis following intra-ocular surgery is a rare but devastating complication. Overall rates are quoted as low as $0.039 \%$ following pars plana vitrectomy [27]. Several studies have implicated sutureless techniques with an increased incidence $[10,28]$, although the relationship is not necessarily directly causal. We have already outlined how wound integrity can be achieved with the correct configuration, placement and suturing of sclerotomies and the use of fluid-air exchange. In the two large studies reporting an increased incidence of endophthalmitis fluid-air exchange was not carried out [10, 26]. In our study there were no cases of endophthalmitis in either group. It is however, difficult to draw conclusions about a rare complication from our small study group. Such a study would require significantly greater numbers [29] and is beyond the scope of our paper.

Overall outcomes were similar for both the 23- and 20gauge groups, including gain in visual acuity, macular hole closure rates and the requirement for further surgery. The rate of post-operative retinal detachment was low in our study. These results are comparable with similar studies in the literature [14-16,30] and suggest that 23-gauge surgery is both safe and effective when compared to gold-standard 20 -gauge vitrectomy.

\section{CONCLUSION}

The purpose of this study was to compare the incidence of extreme changes in post-operative IOP in conventional sutured 20-gauge surgery with that of sutureless 23-gauge vitrectomy in the early post-operative period. We have shown that post-operative hypotony is not more common following 23-gauge surgery complete with fluid air/gas exchange, even with the use of a predominantly sutureless technique. Fluid air/gas exchange is an additional step that is associated with very low risk of post-operative hypotony.

The incidence and magnitude of elevated post-operative IOP was lower in the 23-gauge group when compared to increases in IOP following 20 -gauge vitrectomy. We conclude that 23-gauge vitrectomy is both a safe and effective technique that, in addition to offering many benefits to patients, is associated with less extreme fluctuations in post-operative IOP than conventional 20gauge vitrectomy.

\section{CONFLICT OF INTEREST}

Emily Gosse, Peter Hall and Jonathan Lochhead declare no potential conflict of interest. Richard Newsom has been a speaker for Alcon and has received educational grants from them.

\section{ACKNOWLEDGMENTS}

Declared none.

\section{REFERENCES}

[1] Recchia FM, Scott IU, Brown GC, Brown MM, Ho AC, Ip MS. Small-gauge pars plana vitrectomy: a report by the American Academy of Ophthalmology. Ophthalmology 2010; 117(9): 18517.

[2] Chen JC. Sutureless pars plana vitrectomy through self-sealing sclerotomies. Arch Ophthalmol 1996; 114: 1273-5.

[3] Fujii GY, de Juan Jr E, HumayunMS, et al. Initial experience using the transconjunctival sutureless vitrectomy system for vitreoretinal surgery. Ophthalmology 2002; 109: 1814-20.

[4] Eckardt C. Transconjunctival sutureless 23-gauge vitrectomy. Retina 2005; 25: 208-11.

[5] R Development Core Team. R: A language and environment for statistical computing. R Foundation for Statistical Computing: Vienna, Austria 2009.

[6] Anderson NG, Fineman MS, Brown GC. Incidence of intraocular pressure spike and other adverse events after vitreoretinal surgery. Ophthalmology 2006; 113(1): 42-7.

[7] O'Reilly P, Beatty S. Transconjunctival sutureless vitrectomy: Initial experience and surgical tips. Eye 2007; 21: 518-21.

[8] Haas A, Seidel G, Steinbrugger I, et al. Twenty-three-gauge and 20 -gauge vitrectomy in epiretinal membrane surgery. Retina 2010; 30(1): 112-6.

[9] Gupta OP, Maguire JI, Eagle RC Jr, Garg SJ, Gonye GE. The competency of pars plana vitrectomy incisions: a comparative histologic and spectrophotometric analysis. Am J Ophthalmol 2009; 147(2): 243-50.

[10] Scott IU, Flynn HW Jr, Dev S, et al. Endophthalmitis after 25gauge and 20-gauge pars plana vitrectomy: incidence and outcomes. Retina 2008; 28(1): 138-42.

[11] Parolini B, Prigione G, Romanelli F, Cereda MG, Sartore M, Pertile G. Postoperative complications and intraocular pressure in 943 consecutive cases of 23-gauge transconjunctival pars plana vitrectomy with 1-year follow-up. Retina 2010; 30(1): 107-11.

[12] Nagpal M, Wartikar S, Nagpal K. Comparison of clinical outcomes and wound dynamics of sclerotomy ports of 20, 25, and 23 gauge vitrectomy. Retina 2009; 29(2): 225-31.

[13] Schweitzer C, Delyfer MN, Colin J, Korobelnik JF. 23-Gauge transconjunctival sutureless pars plana vitrectomy: results of a prospective study. Eye 2009; 23(12): 2206-14.

[14] Fine HF, Iranmanesh R, Iturralde D, Spaide RF. Outcomes of 77 consecutive cases of 23-gauge transconjunctival vitrectomy surgery for posterior segment disease. Ophthalmology 2007; 114(6): 1197200.

[15] Lott MN, Manning MH, Singh J, Zhang H, Singh H, Marcus DM. 23-gauge vitrectomy in 100 eyes: short-term visual outcomes and complications. Retina 2008; 28(9): 1193-200.

[16] Misra A, Ho-Yen G, Burton RL. 23-gauge sutureless vitrectomy and 20-gauge vitrectomy: a case series comparison. Eye 2009; 23(5): 1187-91.

[17] Ahn SJ, Woo SJ, Ahn J, Park KH. Comparison of postoperative intraocular pressure changes between 23-gauge transconjunctival sutureless vitrectomy and conventional 20 -gauge vitrectomy. Eye 2012; 26(6): 796-802.

[18] Akçay Bİ, Uyar OM, Akkan F, Eltutar K. Outcomes of 23-gauge pars plana vitrectomy in vitreoretinal diseases. Clin Ophthalmol 2011; 5: 1771-6.

[19] Chieh JJ, Rogers AH, Wiegand TW, Baumal CR, Reichel E, Duker JS. Short-term safety of 23-gauge single-step transconjunctival vitrectomy surgery. Retina 2009; 29(10): 1486-90.

[20] Shimozono M, Oishi A, Kimakura H, Kimakura M, Kurimoto Y. Three-step incision for 23-gauge vitrectomy reduces postoperative hypotony compared with an oblique incision. Ophthalmic Surg Lasers Imaging 2011; 42(1): 20-5.

[21] Chen D, Lian Y, Cui L, Lu F, Ke Z, Song Z. Sutureless vitrectomy incision architecture in the immediate postoperative period evaluated in vivo using optical coherence tomography. Ophthalmology 2010; 117(10): 2003-9.

[22] Rizzo S, Genovesi-Ebert F, Vento A, Miniaci S, Cresti F, Palla M. Modified incision in 25-gauge vitrectomy in the creation of a tunneled airtight sclerotomy: an ultrabiomicroscopic study. Graefes Arch Clin Exp Ophthalmol 2007; 245: 1281-8. 
[23] Ho LY, Garretson BR, Ranchod TM, et al. Study of intraocular pressure after 23-gauge and 25-gauge pars plana vitrectomy randomised to fluid versus air fill. Retina 2011; 31(6): 1109-17.

[24] Yamane S, Kadonosono K, Inoue M, et al. Effect of intravitreal gas tamponade for sutureless vitrectomy wounds: three dimensional corneal and anterior segment optical coherence tomography study. Retina 2011; 31(4): 702-6.

[25] Singh CN, Iezzi R, Mahmoud TH. Intraocular pressure instability after 23-gauge vitrectomy. Retina 2010; 30(4): 629-34.

[26] Framme C, Klotz S, Wolf-Schnurrbusch UE, Wiedemann P, Wolf S. Intraocular pressure changes following $20 \mathrm{G}$ pars-plana vitrectomy. Acta Ophthalmol 2012; 90(8): 744-9.
Eifrig CW, Scott IU, Flynn HW, Jr, Smiddy WE, Newton J. Endophthalmitis after pars plana vitrectomy: Incidence, causative organisms and visual acuity outcomex. Am J Ophthalmol 2004; 138: 799-802.

[28] Kunimoto DY, Kaiser RS. Incidence of endophthalmitis after 20and 25-gauge vitrectomy. Ophthalmology 2007; 114: 2133-7.

[29] Martidis A, Chang TS. Sutureless 25-gauge vitrectomy: risky or rewarding? Ophthalmology 2007; 114: 2131-2.

[30] Ramkissoon YD, Aslam SA, Shah SP, Wong SC, Sullivan PM. Risk of iatrogenic peripheral retinal breaks in 20-G pars plana vitrectomy. Ophthalmology 2010; 117(9): 1825-30.

(C) Gosse et al.; Licensee Bentham Open.

This is an open access article licensed under the terms of the Creative Commons Attribution Non-Commercial License (http://creativecommons.org/licenses/by$\mathrm{nc} / 3.0 /$ ) which permits unrestricted, non-commercial use, distribution and reproduction in any medium, provided the work is properly cited. 